\title{
The dark matter density problem in massive disk galaxies
}

\author{
Benjamin J. Weiner \\ UCO/Lick Observatory, University of California, Santa Cruz, Santa \\ Cruz, CA 95064, USA
}

\begin{abstract}
We discuss measurements of disk mass from non-circular streaming motions of gas in the barred galaxies NGC 3095 and NGC 4123. In these galaxies with strong shocks and non-circular motions, the inner regions must be disk-dominated to reproduce the shocks. This requires dark matter halos of low central density and low concentration, compared to LCDM halo predictions. In addition, the baryonic collapse to a disk should have compressed the halo and increased the dark matter density, which sharpens the disagreement. One possible resolution is a substantial amount of angular momentum transfer from disk to halo, but this is not particularly attractive nor elegant.
\end{abstract}

\section{Introduction}

Schemes for galaxy formation which model the collapse of baryons within a dark matter halo can make useful predictions for the structure of disk galaxies (e.g. Fall \& Efstathiou 1980, Dalcanton et al. 1997, Mo et al. 1998). Conversely, measurements of disk galaxy properties should yield information about the formation process. For example, a success of the Fall \& Efstathiou picture is that it produces disks with the right scalelengths, from the distribution of primordial spins combined with the assumption that the baryons conserve angular momentum during collapse. Cosmological $N$-body and hydrodynamical modelers are now capable of simulating disk galaxy formation with varying degrees of succcess (e.g. Navarro \& Steinmetz 2000). Simulating baryonic dissipation and star formation is still quite uncertain, but $N$-body models make predictions for dark matter halo density and radial profile.

Measurements of the radial distribution of luminous and dark mass in disk galaxies are potentially an important test of disk formation models. However, there is a degeneracy between the luminous and dark matter contributions to the galaxy rotation curve, the "maximum disk" problem (e.g. van Albada et al. 1985). Several workers have approached this problem by studying dwarf and low surface brightness galaxies, where the dark matter is expected to dominate the rotation curve. This line of inquiry has concentrated on the value of the inner slope of the DM halo density profile. Whether the measurements show a $\rho=$ constant core or a $\rho \propto r^{-1}$ cusp remains controversial, e.g. de Blok et al. (2001), Swaters et al. (2003), and several contributions at this conference, and even $N$-body models have disagreed on predictions for the inner slope. 

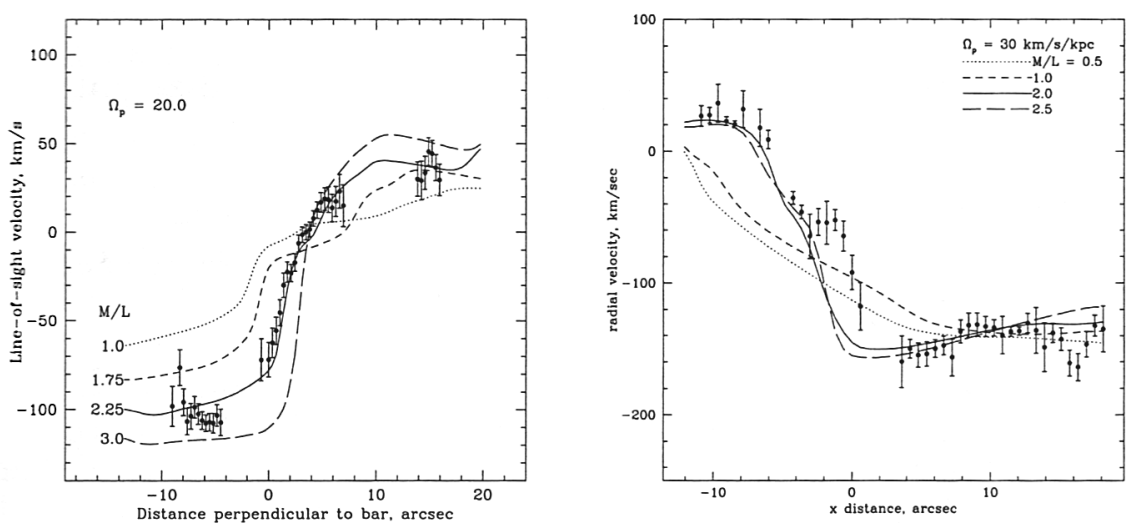

Figure 1. Velocity jumps across the bar shocks in NGC 4123 (left) and NGC 3095 (right), showing Fabry-Perot data (points) and models for various disk $M / L$ (lines).

I describe an alternative way to measure galaxy structure and test formation models, using the internal non-circular kinematics of HSB barred galaxies to determine the contribution of luminous mass and constrain the properties of their dark halos. These results do not constrain the "core/cusp" inner slope issue, which in any case represents a disagreement over the distribution of a very small percentage of dark halo mass, but constrain the dark matter mass within the optical disk of the galaxy.

\section{Barred galaxies, non-circular motions, and disk mass}

The velocity fields of barred galaxies contain additional information beyond the overall rotation curve, which we may use to break the disk-halo degeneracy. Their gas velocity fields commonly have non-circular streaming motions and shocks inside the bar. These motions are caused by the elongated potential of the stellar bar; the dark halo is usually presumed to be dynamically hot and must be rounder than the bar. The strength of the non-circular motions is dependent on the ratio of disk to halo mass within the radius.

The gas flows contain shocks, so a full fluid-dynamical treatmeant is necessary to model the gas velocity field. Our goal is to constrain disk mass by comparing gas-dynamical grid code simulations to high-resolution 2-D velocity fields from Fabry-Perot $\mathrm{H} \alpha$ observations. The full procedure is described in Weiner et al. (2001). We run many models for different values of the parameters stellar disk $M / L_{I}$ and bar pattern speed $\Omega_{p}$; we construct the potential using $I$-band photometry for the stellar disk, and adding the dark halo needed to maintain consistency with the overall rotation curve. We then compare the model velocity fields to observations inside the bar radius, to constrain disk $M / L_{I}$ and bar $\Omega_{p}$. Given the measurement of disk $M / L$, the dark halo contribution to the rotation curve is also measured. 
Disk $\mathrm{M} / \mathrm{L}=2.25, \quad$ NFW halo $\mathrm{V}_{200}=87.51, \mathrm{c}=3.23$, chisq $=1.02$

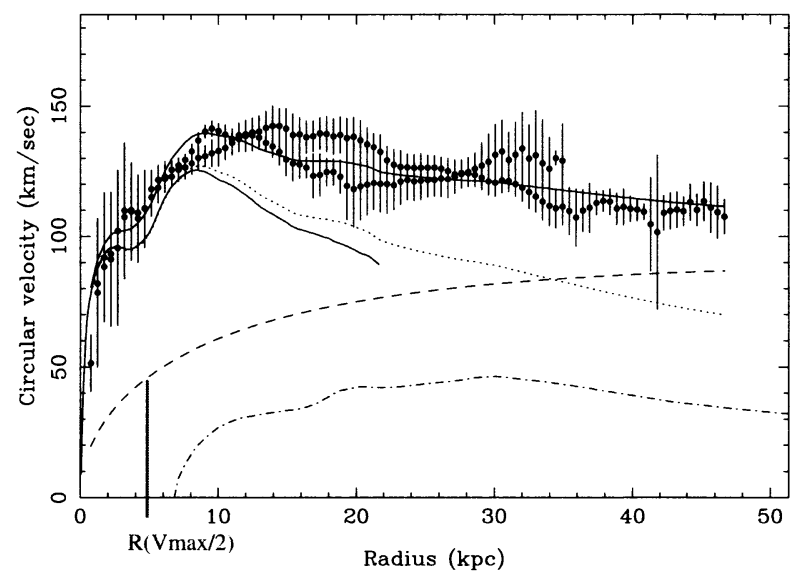

Figure 2. NGC $4123 \mathrm{HI}$ rotation curve with stellar disk (solid), HI gas (dot-dash), stars+gas (dotted), and dark halo (dashed) contributions for disk $M / L=2.25$. The fiducial radius $R\left(V_{\max }\right) / 2$ ) of the dark halo is indicated.

Figure 1 shows, for the barred galaxies NGC 4123 and NGC 3095, a cut through the data and models which crosses the bar shock. There is a sharp jump in velocity across the bar. We plot several models to show the effect of varying disk $M / L$ while keeping pattern speed constant. As $M / L$ increases, the bar influence, non-circular motions, and shocks become stronger. For $M / L_{I} \leq 1$ the shock is minimal. A relatively high disk $M / L_{I}$ is needed to produce the observed shocks, 2.25 in NGC 4123 and 2.0 in NGC 3095. The fit for NGC 3095 is not perfect, but the sense is clear - low mass disks cannot make a strong enough shock. Comparisons over the full 2-D velocity field inside the bar bear this out (Weiner et al. 2001 for NGC 4123). The comparison over the full range of model parameters also shows that a fast-rotating bar with a high pattern speed $\Omega_{p}$ is required.

\section{Dark matter halo densities}

Given the measurement of disk $M / L$, we can return to the rotation curve to determine the best fitting dark halo. Figure 2 shows a fit to the HI rotation curve of NGC 4123 for the measured disk $M / L_{I}=2.25$. The bar radius is 5 $\mathrm{kpc}$, so the disk $M / L$ is constrained only inside that radius by our modeling. However, at $5 \mathrm{kpc}$ the disk contribution to the rotation curve is already near its maximum; any falloff in $M / L$ outside $5 \mathrm{kpc}$ has minimal effect. This galaxy has $R_{25}=11 \mathrm{kpc}$ and inside the optical disk the stars dominate the rotation curve; it is very close to maximum disk. NGC 3095, which is larger in $V_{\text {circ }}$ and radius, is also close to maximum disk.

Because these galaxies are dominated by stars in the center, we cannot distinguish between different values of the inner slope of the DM density profile. 


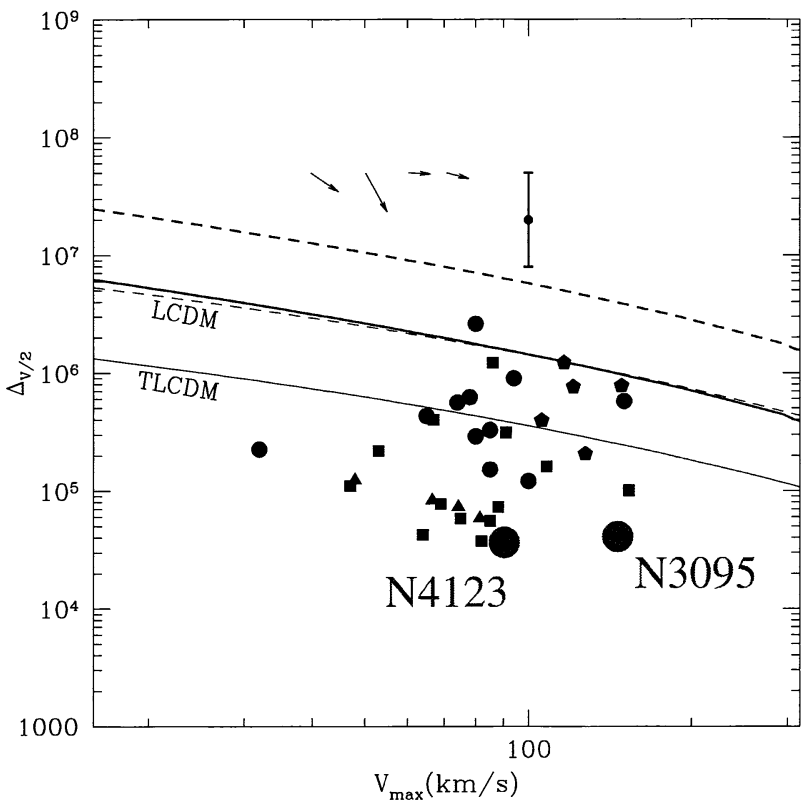

Figure 3. $\Delta\left(V_{\max } / 2\right)$, mean DM density within $R\left(V_{\max } / 2\right)$, versus $V_{\text {max }}$, from Alam et al. (2002). Small points are upper limits from LSB galaxies, curves are LCDM and tilted LCDM models. The DM densities for NGC 4123 and NGC 3095 are overplotted (large points). These fall well below the LSB galaxies.

However, we can put strong constraints on the amount of dark matter within a given radius. Alam et al. (2002) emphasize that it is easier to make a robust measure of the mean DM density within a fiducial radius than to measure the slope of a density profile, since mean density $\Delta \propto M(<R) / R^{3} \propto(V / R)^{2}$. They use the radius $R\left(V_{\max } / 2\right)$ where the DM rotation curve reaches half-maximum velocity and compute the mean enclosed DM density $\Delta\left(V_{\max } / 2\right)$ in units of the critical density.

Figure 2 indicates the location of $R\left(V_{\max } / 2\right)$ for NGC 4123 , at $\sim 5 \mathrm{kpc}$. Because the stars dominate the inner rotation curve, $R\left(V_{\max } / 2\right)$ is relatively large. Equivalently the halo has a large core or scale radius, and thus a low concentration $c_{N F W}=3.2$, lower than predicted from LCDM simulations (Bullock et al. 2001, see Weiner et al. 2001). The rotation curve fit can be tweaked to make the DM halo curve rise more sharply, but the amount of DM within 5-10 kpc is limited, and the DM must have a longer scale length than the disk; there is not room for much dark matter within a few scalelengths of the optical disk.

Figure 3 reproduces Figure 2 of Alam et al. (2002), showing measurements of $\Delta\left(V_{\max } / 2\right)$ versus $V_{\max }$ for a sample of LSB galaxy rotation curves, and theoretical predictions from fitting DM halos in LCDM and tilted LCDM simulations. The LSB points are upper limits since baryons may contribute slightly to the LSB rotation curves. I have overplotted the mean densities $\Delta$ for the dark halos of the barred galaxies NGC 4123 and NGC 3095. 
The DM densities of the HSB barred galaxies are well below the LSB points and far below the LCDM and TLCDM predictions. The disagreement is sharpened because the predictions are for DM-only systems, pre-baryonic collapse, while the HSB measurements are of post-collapse halos. As baryons collapse into an angular momentum supported disk, they move inward and should also draw the dark matter halo inward. This process is commonly modeled with the "adiabatic compression" assumption, which includes detailed conservation of angular momentum for the dark matter (Blumenthal et al. 1986).

It is extremely difficult to understand how the post-collapse halo could be less concentrated than the pre-collapse state. In fact, when we attempted to reverse the adiabatic compression, running the collapse backwards for NGC 4123, we obtained at best an implausible, constant density initial state and at worst a simply unphysical initial density profile (Weiner et al. 2001). We concluded that the assumptions of adiabatic compression must be violated in order to produce the galaxy seen today.

\section{Conclusions}

In the two HSB barred galaxies we have modeled, the luminous disk dominates the mass within several scalelengths, requiring a low central density for the dark matter halo. A similar conclusion holds for the Milky Way based on its noncircular motions and on microlensing (Gerhard, this meeting). The measured post-collapse dark halo densities are lower than predicted pre-collapse halo densities, even though the collapse process should drive the DM density higher.

Two inexplicable galaxies are bad enough, but this is also a generic problem for HSB galaxies if the stellar disk dominates inside a few scale lengths. The question of whether disk galaxies are luminous or DM dominated has a long history. Recently, based on the weak correlation of Tully-Fisher residuals with size and surface brightness, Courteau \& Rix (1999) have argued that disk galaxies are very DM dominated, but our results, microlensing studies of the Milky Way, and the ubiquity of $m=2$ spirals and bars (Athanassoula et al. 1987) suggest that luminous matter must be important inside a few scalelengths. Since we don't yet understand how the disk and halo are coupled in the formation process to make the TF relation independent of surface brightness, it is not certain what the TF residuals should show. Kranz et al. (2003) have modeled spiral arm streaming motions, and suggest that galaxies with $V_{c}>200 \mathrm{~km} / \mathrm{sec}$ are close to maximum disk, while below that galaxies are DM dominated. (I speculate that DM-dominance depends on surface brightness; NGC 4123 is luminous matter dominated, has a low $V_{c}=135 \mathrm{~km} / \mathrm{sec}$, but is quite HSB.)

Are the disk-dominated NGC 4123 and NGC 3095 likely to be representative of HSB galaxies? The $M / L_{I}$ ratios we find for NGC 4123 and NGC 3095 are reasonable for their colours, compared to the models of Bell \& de Jong (2001). The galaxies are on the $I$-band Tully-Fisher relation (Giovanelli et al. 1997). Barred and unbarred galaxies are on the same $\mathrm{TF}$ relation and should have similar halo properties (Courteau et al. 2003). It seems unlikely that galaxies of similar colours and surface brightnesses could have very different $M / L$ and inhabit the same TF relation. 
How can the low-density dark halos we find be reconciled with the expectations of CDM? It is possible to modify the initial power spectrum to lower the power on small scales, but probably not more than the TLCDM model shown in Figure 3. Even that does not explain why the post-collapse HSB galaxy halos are less dense than the pre-collapse predictions or LSB upper limits.

Under the assumption of adiabatic compression, the baryons always tend to drag the DM inward, and it is difficult to keep the halo's scale length much greater than the disk's. Two potential mechanisms are (1) blowout of baryons, which could loosen the binding of the DM, and (2) transfer of angular momentum from baryons to DM. It is not clear that blowout is effective in galaxies with high $V_{c}$. Angular momentum transfer is potentially very effective, but unfortunately may upset the elegant paradigm for disk formation developed since Fall \& Efstathiou (1980); for example, if the baryons lose a significant amount of angular momentum, disk sizes may no longer be predicted naturally.

In conclusion I suggest that we should not regard observations of galaxy structure as merely tests of which, if any, CDM model is correct, but as probes of the astrophysics of galaxy formation. Progress in this field may come as we move beyond comparing CDM-only halos to observed galaxies; real galaxies have baryons, and we need a better understanding of what happens during the collapse phase of disk formation.

Acknowledgments. I thank the American Astronomical Society for a travel grant which allowed me to attend this conference.

\section{References}

Alam, S.M.K., Bullock, J.S., \& Weinberg, D.H. 2002, ApJ, 572, 34

Athanassoula, E., Bosma, A., \& Papaioannou, S. 1987, A\&A, 179, 23

Bell, E.F., \& de Jong, R.S. 2001, ApJ, 550, 212

Blumenthal, G.R., Faber, S.M., Flores, R., \& Primack, J.R. 1986, ApJ, 301, 27

Bullock, J.S. et al. 2001, MNRAS, 321, 559

Courteau, S., \& Rix, H.-W. 1999, ApJ, 513, 561

Courteau, S., Andersen, D.R., Bershady, M.A., MacArthur, L.A., \& Rix, H. 2003, ApJ, 594, 208

Dalcanton, J.J., Spergel, D.N., \& Summers, F.J. 1997, ApJ, 482, 659

de Blok, W.J.G., McGaugh, S.S., \& Rubin, V.C. 2001, AJ, 122, 2396

Fall, S.M., \& Efstathiou, G. 1980, MNRAS, 193, 189

Giovanelli, R. et al. 1997, AJ, 113, 53

Kranz, T., Slyz, A., \& Rix, H. 2003, ApJ, 586, 143

Mo, H.J., Mao, S., \& White, S.D.M. 1998, MNRAS, 295, 319

Navarro, J.F., \& Steinmetz, M. 2000, ApJ, 538, 477

Swaters, R.A., Madore, B.F., van den Bosch, F.C., \& Balcells, M. 2003, ApJ, 583,732

van Albada, T.S., Bahcall, J.N., Begeman, K., \& Sancisi, R. 1985, ApJ, 295, 305

Weiner, B.J., Sellwood, J.A. \& Williams, T.B. 2001, ApJ, 546, 931 